\title{
DISEÑO DE MAPEO DE LA CADENA DE VALOR ÁREA DE PRODUCCIÓN CHANCLA LÁMINA EVA EN LA EMPRESA DJ-GROUP
}

\section{MAPPING DESIGN OF THE VALUE CHAIN PRODUCTION AREA CHANCLA EVA SHEET IN THE COMPANY DJ-GROUP}

Yaritza Jaramillo Barona, Richard Fernando Urbano Rayo y Carlos Andrés Nieto

Semillero Simec

Institución Universitaria Antonio José Camacho

Cómo citar este artículo:

Para citar este artículo: Jaramillo, B. Y., Urbano, R.F. y Nieto, C.A. (2021). Diseño de mapeo de la cadena de valor área de producción chancla lamina Eva en la empresa dj-group). Revista Sapientia, 13(26), 51-59.

\section{RESUMEN}

El presenta artículo tiene como objetivo un análisis de proceso en la empresa de calzado Dj-Group, dedicada a la fabricación de chancla a base de lámina EVA. Una vez identificado el problema que presenta en cada una de las áreas, se evidencian desperdicios y desplazamientos repetitivos en la línea masculina. El estudio se realizó a partir de un VSM (Mapeo de la Cadena de Valor, según sus siglas en inglés), una herramienta utilizada en la industria para realizar diagnóstico de todas las actividades del proceso que identifica problemas y oportunidades de mejora, para esto se lleva a cabo un análisis de todas las variables que componen el proceso desde el proveedor de la materia prima hasta la entrega al cliente. Los resultados permitieron identificar que la empresa Dj-Group cuenta con dos tipos de desperdicios en la línea de producción del calzado masculino: tanto en desplazamiento repetitivo como en tiempos improductivos; al solucionar los problemas encontrados se evidenció la disminución del costo por par de chanchas o incluso la disminución de tiempo en la fabricación de chanclas que permita una mayor efectividad.

\section{PALABRAS CLAVE}

VSM, desplazamiento, desperdicios, lámina Eva, efectividad.

\section{ABSTRACT}

The objective of the present article is a process analysis in the Dj-Group footwear company, dedicated to the manufacture of EVA foil-based flip flops. Once the problem that it presents in each of the areas has been identified, waste and repetitive displacements are evident in the male line. The study was carried out from a VSM (Value Chain Mapping, according to its acronym in English), a tool used in the industry to diagnose all the activities of the process that identifies problems and opportunities for improvement. carries out an analysis of all the variables that make up the process from the raw material supplier to delivery to the customer. The results allowed identifying that the Dj-Group company has two types of waste in the production line of men's footwear: both in repetitive displacement and in unproductive times; When solving the problems found, a decrease in the cost per pair of slides was evidenced, or even a decrease in time in the manufacture of flip-flops that allows greater effectiveness.

\section{KEYWORDS}

VSM, displacement, waste, Eva lamina, effectiveness. 


\section{2 | Sapıentia Vol. 13, №. 26, noviembre 202l. pág. 51-59 ISSN 1909-08II}

\section{INTRODUCCIÓN}

En la actualidad, el mundo de la industria trata de afinarse cada día en los procesos que rodean las diferentes industrias, esta mejora se puede ver desde diferentes puntos de vista enfocados en el proceso, como por ejemplo: la mejora en la maquinaria daría a entender que la eficiencia en la línea de producción debería de mejorar en tiempos, poco desperdicio y automatización; la mejora en capacitación técnica de los trabajadores conllevaría a un óptimo desempeño en el proceso a nivel individual o en conjunto. Esto entre otros puntos de vista como lo puede ser un mejor software para el seguimiento del producto y mejores instalaciones.

El mapeo del flujo de valor es una técnica de gestión ajustada que ayuda a las empresas a visualizar procesos en un esfuerzo por definir y optimizar los pasos involucrados en la obtención de un producto, servicio o proyecto de valor agregado de principio a fin. Cuando se realiza de manera eficaz, el mapeo del flujo de valor arroja luz sobre las formas de reducir el desperdicio dentro de los procesos o de aumentar los elementos que agregan valor directamente a los clientes (TERRY, 2021).

Respecto a la implementación del VSM, (Guevara, 2007) afirma que:

cuando se realizan las observaciones, los analistas de VSM con frecuencia comienzan a seguir a la gente al momento en que ésta desempeña su trabajo. Pero tal vez no todo lo que el empleado hace, se relaciona directamente con la fabricación y desarrollo del producto que se está estudiando o quizá se deje fuera a otros protagonistas. (Guevara, 2007)

En este sentido, en Colombia, y a pesar de la implementación de iniciativas, la metodología Lean ha registrado un crecimiento discreto. Esta ha sido principalmente adoptada por multinacionales como GM Col motores, Sofasa, Sandford y Belcorp, entre otras, debido a los requerimientos de sus casas matrices y especialmente en la industria automotriz, del papel y del plástico; no obstante, brinda oportunidades de mejoramiento a todo tipo de industria, bien sea de manufactura o servicios (Industria, 2021).

Aunque se presentan dificultades en la implementación y algunos aspectos como el talento humano no es partícipe del $100 \%$ de la fabricación del producto, es necesario tener en cuenta que la aplicación del VSM denota las necesidades directas de la empresa; por otra parte, aunque no alcance a abarcar todos los puntos de manera específica, no quiere decir que la implementación de la herramienta no sea óptima y/o funcional.

Para ejemplificar como referencia en la aplicación de la metodología VSM en la empresa Coservicios S.A. se obtuvo un mejoramiento de la productividad y eficiencia, que finalmente se pudo evidenciar por el cliente con costos, tiempos de entrega más reducidos y mejor calidad con el producto realizado. El porcentaje de tiempo de valor no agregado disminuyó en un 5\%. Se ahorraron 2 kilómetros de recorridos por equipo. El tiempo de Conclusión 136 Ciclo total disminuye 33 horas en el total del equipo estándar analizado, al igual que el tiempo puerta a puerta que también tiene un notable ahorro de 12 días. Hay mayor rotación del inventario, un incremento de 36 vueltas adicionales en cuanto a materia prima y productos en proceso.

De acuerdo a lo anterior, la investigación que refiere el presente artículo se desarrolló en la empresa Dj-Group, línea de producción chancla lámina Eva, ubicada en Acopi Yumbo, permitió identificar las actividades $\mathrm{u}$ operaciones que pueden afectar un flujo continuo en el proceso y que no agregan valor. Esto con el fin de buscar alternativas de mejora e implementación de metodologías de lean manufacturing, mejorando así los costos de producción y generando mayor flujo en los procesos.

A partir de los hallazgos de la investigación se proyecta aumentar la productividad mediante la eliminación de desperdicio y proponer oportunidades de mejora por cada área que influya en el proceso. Para analizar las problemáticas encontradas en la compañía fue necesario mencionar las causas identificadas para la producción de chancla de lámina Eva, que no agregan valor, generando improductividad en las áreas e incumplimi- 
-ento miento de tiempos disponibles tanto de maquinaria y como de operación. Es así como se identificó alta acumulación y reproceso del material por mal manejo en las áreas, desorden y riesgo en las diversas etapas del proceso productivo, pérdida de tiempo para ubicar los materiales, incluyendo también empaque final y distribución inadecuada que impide un flujo continuo en los procesos.

En el desarrollo de la estrategia de VSM se evidencia que existen falencias, frente a la cuales es posible crear mejoras con base en herramientas que ayuden a la comprensión de datos.

\section{MARCO TEÓRICO}

El mapa de flujo de valor o Value Stream Mapping (VSM) es un diagrama o mapa que tiene como objetivo visualizar, analizar y mejorar el flujo dentro de un proceso de producción. Este flujo hace referencia a los procesos y la información que se realizan desde el inicio del proceso hasta su entrega al cliente (Marte, 2020).

El Value Stream Mapping es una herramienta visual que permite hacerse una idea de todo el proceso de fabricación de un producto, desde la recepción de la mercancía, pasando por los procesos de fabricación y almacenaje, hasta la entrega final del cliente (Marte, 2020).

Según John Shook la frase "flujo de valor" trata de concentrarse en atención infinita a establecer el flujo, eliminar el desperdicio y agregar valor. A lo largo de las últimas décadas se ha implementado en diferentes medios industriales, por ejemplo, los profesionales del sistema de producción de Toyota lo utilizan para representar estados actuales y futuros, o "ideales" en el proceso de desarrollo de planes de implementación para instalar sistemas ajustados (BAUDIN, ¿De dónde provienen los "Value Stream Maps"?, 2013).

Por otro lado, el ingeniero japonés Shigeo Shingo de la compañía Toyota, ideo y desarrollo una serie de etapas para reducir los tiempos de cambio de producto al evidenciar tiempos superiores de 4 horas en la línea de estampado. Aunque en la definición de SMED se menciona una reducción de cambios o puesta a punto menores de 10 minutos, esto no siempre podrá lograrse. Sin embargo, a la fecha un tiempo de cambio de troqueles menor a 100 segundos, o de un toque o paso, es considerado un "changeover" de clase mundial (Clockwork, 2020).

En Colombia, y a pesar de la implementación de iniciativas, lean ha registrado un crecimiento discreto. Esta metodología ha sido principalmente adoptada por multinacionales como GM Colmotores, Sofasa, Sandford y Belcorp, entre otras; debido a los requerimientos de sus casas matrices y especialmente en la industria automotriz, del papel y del plástico; no obstante, brinda oportunidades de mejoramiento a todo tipo de industria, bien sea de manufactura o servicios (Industria, 2021).

Según (Yépez, 2017), se revisaron los principales métodos usados lean a través de una matriz comparativa, la cual indica que de acuerdo con el presupuesto demandado para la implementación de estas herramientas muy pocas empresas lo realizan:

en el contexto colombiano, pocas empresas realizan la implementación de lean de manera exitosa (Arrieta, Botero y Romano, 2010). Uno de los problemas fundamentales es la falta de metodologías de implementación práctica de la filosofía lean, lo cual también se evidencia en el mundo. En segundo término, los tiempos de implementación de lean manufacturing pueden ser de pocos meses o incluso de años (Fortuny-Santos, Cuatrecasas Arbós, Cuatrecasas-Castellsaques y Olivella-Nadal, 2008), lo cual implicaría inversiones adicionales que impactan los presupuestos de estas empresas y por ello el nivel de implementación de lean manufacturing es reducido. 


\begin{tabular}{|c|c|c|c|}
\hline Título & Objetivo & $\begin{array}{c}\text { Herramienta } \\
\text { utilizizada }\end{array}$ & Resultados obtenidos \\
\hline $\begin{array}{l}\text { "Saint gobain perfomace } \\
\text { plastics: modelo lean a } \\
\text { través de VSM" Fuente } \\
\text { especificada no } \\
\text { válida. }\end{array}$ & $\begin{array}{l}\text { Identificar el valor y } \\
\text { optimizar los } \\
\text { procesos. } \\
\text { Beneficios: } \\
\text { Conocimiento del } \\
\text { aporte de valor y } \\
\text { del valor } \\
\text { inmovilizado }\end{array}$ & $\begin{array}{ll}- & 5 S \\
: & \text { KAIZEN } \\
: & \text { KANBAN } \\
- & \text { VSM }\end{array}$ & $\begin{array}{l}\text { - Conocimiento del lead time, } \\
\text { como requisito para una correcta } \\
\text { implantación del futuro sistema } \\
\text { de monitorización de la } \\
\text { producción. }\end{array}$ \\
\hline $\begin{array}{l}\text { Productos MESA: } \\
\text { Implementación de } \\
\text { Modelo lean a través } \\
\text { de VSM Fuente } \\
\text { especificada no } \\
\text { válida. }\end{array}$ & $\begin{array}{c}\text { Pivotar el modelo } \\
\text { productivo hacia } \\
\text { un estándar LEAN. } \\
\text { Beneficio: } \\
\text { Identificar el valor } \\
\text { y optimizar } \\
\text { procesos, así } \\
\text { como mejora de la } \\
\text { imagen de planta. }\end{array}$ & $\begin{array}{ll}- & 5 S \\
- & \text { KAIZEN } \\
- & \text { KANBAN } \\
- & \text { VSM }\end{array}$ & $\begin{array}{l}\text { - La eficiencia del sistema LEAN } \\
\text { - Se conectaron las mejoras de } \\
\text { una manera interdisciplinar. } \\
\text { - Se redujo el desperdicio y los } \\
\text { costes. }\end{array}$ \\
\hline
\end{tabular}

Tabla 1. Empresas que han implementado la herramienta VSM y sus resultados

Fuente: elaboración propia.

\section{METODOLOGÍA}

La efectividad es un tema de vital importancia en la industria, a través de esta se visualiza el estado del proceso para indicarnos qué tan productivo puede estar. Con la ayuda de indicadores o variables de medición se puede incluso llegar a obtener mejoras productivas en pro de la mejora de una industria. La empresa Dj-Group, ubicada en Yumbo, Valle del Cauca, con una trayectoria de seis años en el sector calzado, dedicada a la producción y comercialización de chanclas lámina EVA con un alcance de producción en las líneas de hombre, mujer y niñ@s, ha requerido conocer las fallas que se pueden presentar en la fabricación en uno de sus productos para elaborar una mejora continua en cada proceso.

Para poder realizar un análisis en la producción de línea hombre es necesario conocer que existen varias herramientas enfocadas en este objetivo, las cuales en el transcurso del tiempo han demostrado ser eficaces al momento de su implementación, en este caso se ha optado por el VSM (Value Stream Mapping).

Para poner en práctica la herramienta VSM, es fundamental contar con un equipo interno capacitado, que conozca cada paso del proceso y así generar un criterio constructivo, que genere datos exactos de cada acción que se toma para la transformación de la materia prima. Se deben tomar todos los valores existentes de forma que contengan información precisa, la cual ayuda a comprender cómo es la funcionalidad de cada actividad que se lleva a cabo en la transformación de chancla lámina Eva, en la planta se cuenta con varias áreas de operación como son: Inyectora, Troquelado, Transfer, Perforado, Huellado y Ensamble; en estas la información que se obtiene es minuciosa y detallada, por ende, es necesario tomar tiempos, distancias, número de operarios y cualquier acción que intervenga en la realización del producto.

Para mejorar la interpretación de los datos adquiridos se utilizan métodos gráficos que permiten una mejor ilustración de la información como, por ejemplo: diagrama de flujo, diagrama de espagueti y gráficos de barra. Con la información plasmada en dichas herramientas que permiten una mejor comprensión para el equipo de trabajo, se puede llegar a conclusiones que ayuden a conocer las falencias que a simple vista no se detallan, como pueden ser: tiempos muertos, recorridos innecesarios, tiempos que no agregan valor, entre otros.

Como aspecto importante, y para la implementación de un mapeo en la cadena de valor, el primer paso es obtener la información detallada en el proceso; con el apoyo de estos valores y la herramienta VSM se pueden desplegar variables concluyentes del área a evaluar. Por medio de la ejecución en tablas de análisis por proceso encontramos dichas variables: Cambio de operación (CO), Tiempos de Ciclo (TC) y Otros Tiempos (OT), esta información obtenida es la que al final se implementa en el gráfico VSM. El siguiente ejemplo se ha tomado del área de inyectora:

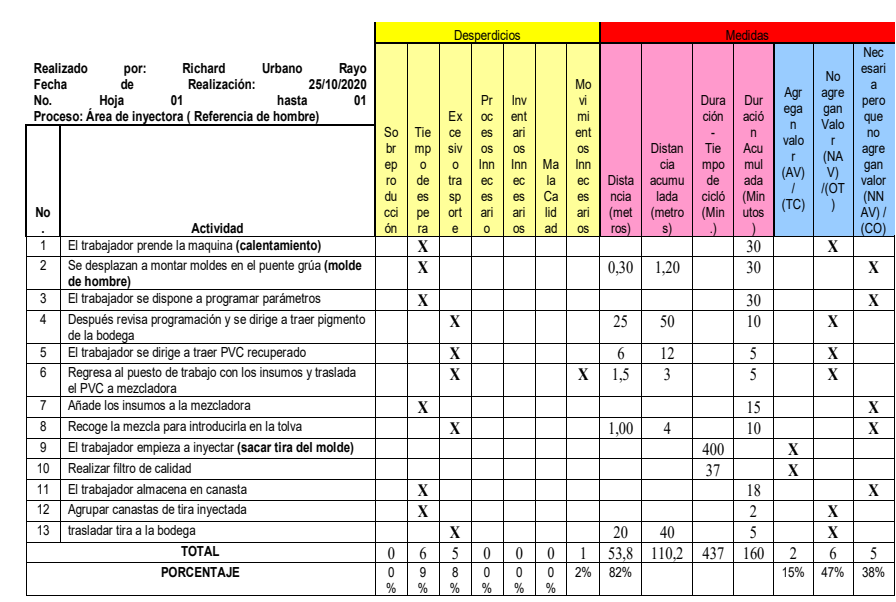

Tabla 2. Análisis área de inyectoria Fuente: elaboración propia. 
Con esta tabla se puede evaluar cada actividad que se realiza en esta área. Mediante la asistencia del equipo de trabajo seleccionado se logra marcar cada acción en un tipo de característica (desperdicio), con la que se saca un valor porcentual que equivale a una parte del proceso, dicho valor se extrae entre el número de casillas marcadas dividido por la suma de los totales. Al terminar todos los diagnósticos, se pueden concluir los tipos de desperdicios con factores significativos que son: tiempos de espera y excesivos transportes. Gracias a la investigación realizada, se puede proceder a la creación del VSM actual de la empresa Dj-Group, teniendo en cuenta los siguientes símbolos:

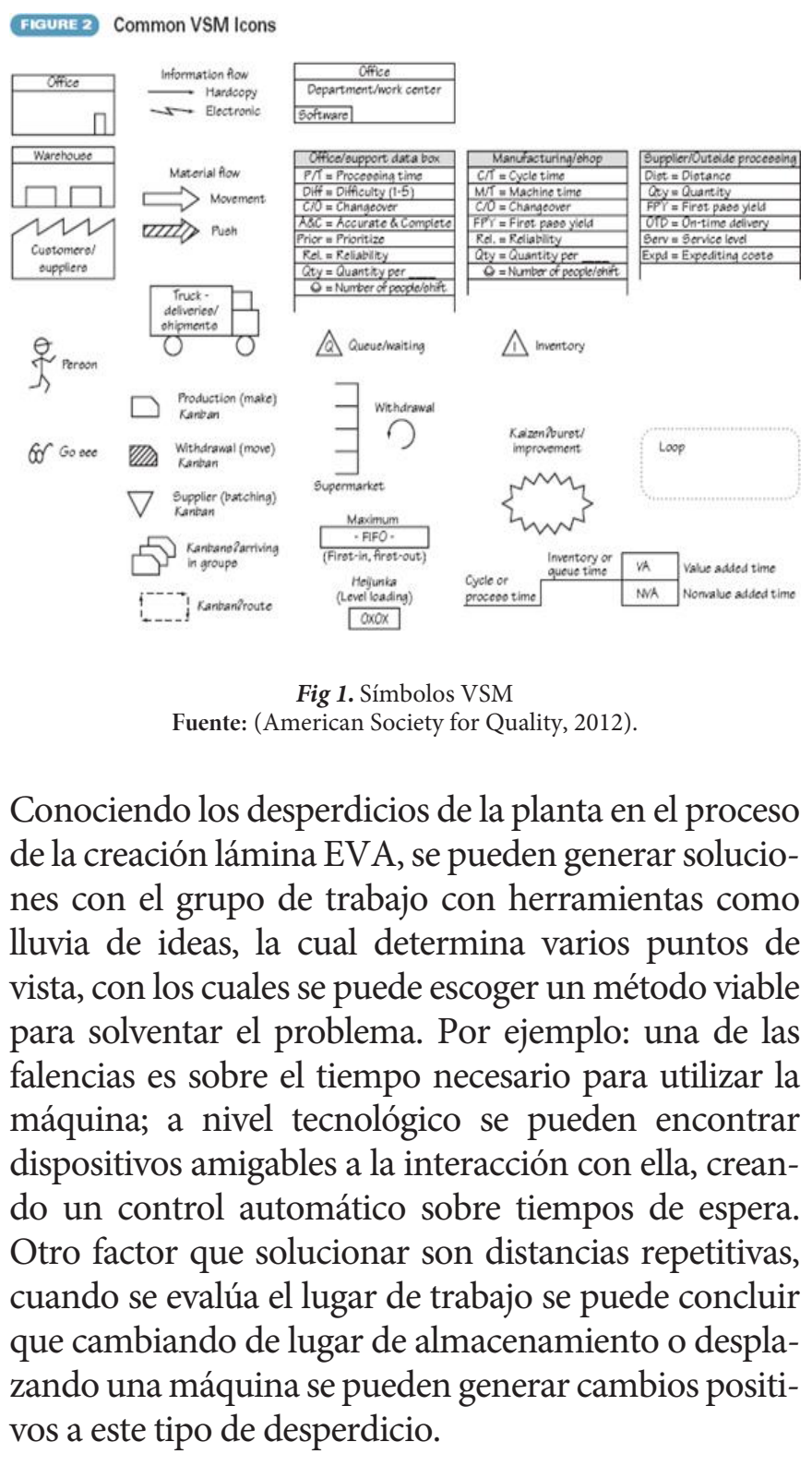

Es necesario analizar nuevamente la efectividad del proceso de acuerdo con las correcciones implementadas y así obtener un nuevo panorama de la eficiencia productiva.

Realizando el seguimiento detallado que se hizo al principio (toma de tiempos de cada proceso), se encuentran datos favorables acerca de los cambios realizados y el aumento de la efectividad de $73.5 \%$ a $76.6 \%$, comprobando que los cambios realizados alcanzaron efectos provechosos frente a los datos anteriormente recolectados; se evidencia una disminución frente a los desperdicios (tiempos y desplazamientos) pasando de lead time de 21.37 a 19.27 horas y un recorrido de 1371 a 1178 metros. Con la reestructuración realizada se evidencia una reducción de costos de par fabricados, pasando de \$3.052 a \$2.897.

\section{RESULTADOS}

Con los hallazgos ya abarcados de manera general mediante el VSM, presentan diferentes falencias las cuales dan como resultado planes de mejoramiento por las diferentes áreas, que se van a especificar a partir de gráficos y tablas.

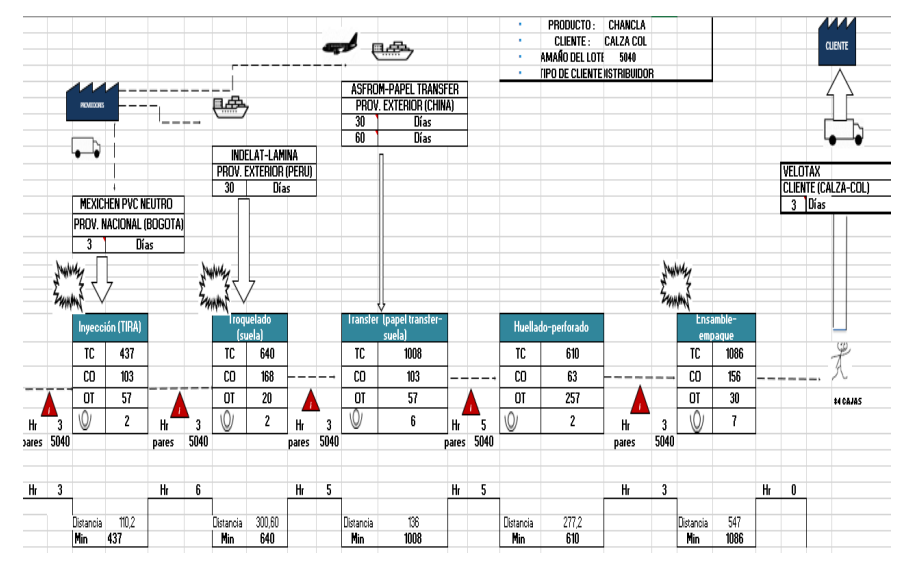

Fig 2. VSM actual de la empresa Dj-Group Fuente: elaboración propia.

De acuerdo con la implementación de la herramienta VSM en la empresa Dj-Group se logra obtener un diagrama con datos concretos del proceso de elaboración de la línea hombre, la cual nos permite encontrar falencias y oportunidades de mejora. 


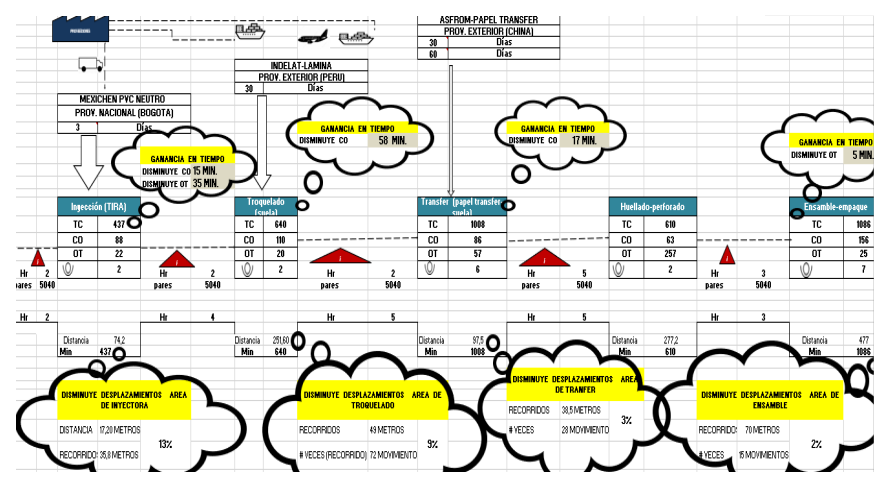

Fig 3. VSM futuro empresa Dj-Group Fuente: elaboración propia

Por medio del VSM actual, donde se encontraron oportunidades de mejora, se pudo obtener un nuevo análisis sobre el proceso, después de realizar acciones correctivas sobre tiempos que no aportan al proceso y desplazamientos repetidos.

\begin{tabular}{|c|c|c|c|c|}
\hline \multicolumn{5}{|c|}{ DJ GROUP COMPANY SA.S } \\
\hline \multicolumn{5}{|c|}{ COMPARATIVO DE COSTO REF. DE HOMBRE } \\
\hline COSTOS DIRECTOS & & ACTUAL & & FUTURO \\
\hline Costo directo de Materiales & $\$$ & $11.273 .567,08$ & $\$$ & $11.094 .360,36$ \\
\hline Costo directo de mano de obra & $\$$ & $3.048 .089,66$ & $\$$ & $2.486 .790,71$ \\
\hline Total directos & $\$$ & $14.321 .656,74$ & $\$$ & 13.581.151,07 \\
\hline Costos indirectos & $\$$ & $1.062 .926,93$ & $\$$ & $1.020 .430,07$ \\
\hline Total horas operativas & & $21,37 \mathrm{hrs}$ & & $19,27 \mathrm{hrs}$ \\
\hline Total de trabajadores & & trabajadores & & trabajadores \\
\hline COSTO TOTAL & & $15.384 .583,66$ & & 14.601 .581 \\
\hline TOTAL PARES & & 5040 & & 5.040 \\
\hline COSTO UNITARIO & $\$$ & 3.052 & & 2.897 \\
\hline REDUCCION COSTO $x$ PAR FABRICADO & $\$$ & & & 155 \\
\hline REDUCCION COSTO X 5040 PARES & $\$$ & & & 783.003 \\
\hline INVERSION TOTAL & $\$$ & & & 2.312 .400 \\
\hline
\end{tabular}

Fig 4. Comparativo de costo/inversión

$$
\text { Fuente: elaboración propia }
$$

Analizando la recolección de datos se puede realizar una comparación en costo/beneficio, entre el VSM actual y el futuro, de la cual se deduce el cambio a nivel monetario que se obtiene después de realizar acciones de mejora como: reducción de recorridos, movimientos, reubicación de operarios y reubicación de máquinas.

\begin{tabular}{|c|c|c|c|}
\hline VSM & $\begin{array}{c}\text { Lead time } \\
\text { (horas) }\end{array}$ & Eficiencia \% & Recorridos metros \\
\hline Actual & 21.37 & $73,5 \%$ & 1371 \\
\hline Futuro & 19.27 & $76.6 \%$ & 1178 \\
\hline
\end{tabular}

Tabla 3. Cuadrado comparativo del (PCE) eficiencia delciclo del proceso Fuente: elaboración propia
A partir de los datos recolectados se concluye que se obtendrá mejora en lead time de 2.10 horas de reducción del tiempo operativo, un aumento en la eficiencia del 3.1\% y un recorte de distancia de 193 metros.

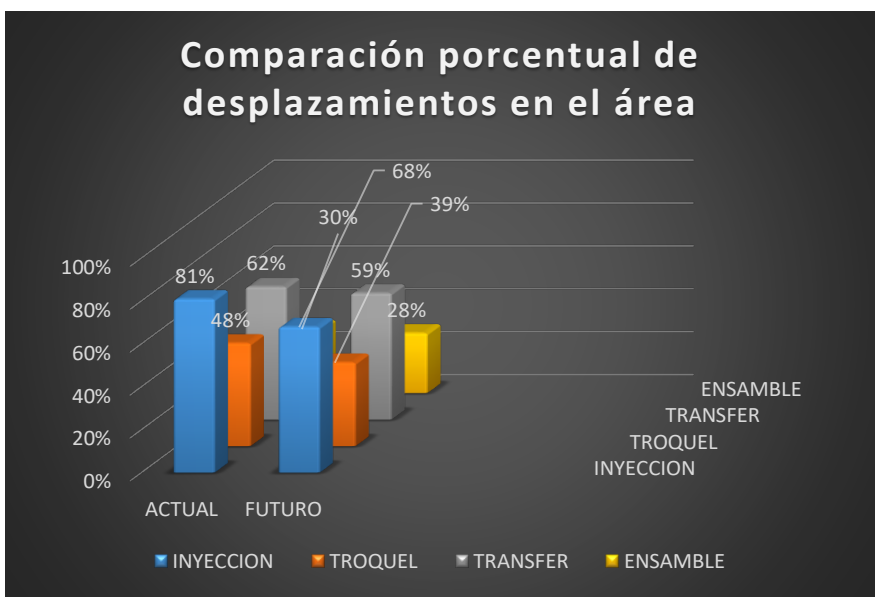

Fig 5. Comparativo de desplazamiento del área Fuente: elaboración propia

En la Figura 4 identificamos de manera porcentual la disminución en desplazamientos del área de inyección con un $13 \%$ y el área de troquelado con un $9 \%$.

De manera general, los resultados que se espera obtener a futuro en la compañía DJ-GROUP de acuerdo a esta investigación equivale a 5.040 pares que equivalen a 84 cajas.

\section{ÁREA DE INYECTORA}

- Desplazamiento disminuye $13 \%$

- Aumento de la eficiencia del $10 \%$ que equivalen a 500 tiras adicionales de fabricación

- Disminuye 17.20 metros de distancia

- Disminuye el total de recorrido a 37 metros

- Ganancia en tiempo de 50 minutos 


\section{ÁREA DE TROQUELADO}

- Desplazamiento disminuye $9 \%$

- Aumento de la eficiencia del $10 \%$ equivalen a 22 docenas adicionales de fabricación

- Disminuye 49 metros de distancia

- Disminuye 72 movimiento (ocasionados por traslado de suela)

- Ganancia en tiempo de 58 minutos

\section{ÁREA DE TRANSFER}

- Desplazamiento disminuye 3\%

- Disminuye 38.5 metros de recorridos

- Disminuye 28 movimientos (ocasionados por traslado de suela)

- Ganancia en tiempo de 17 minutos

\section{ÁREA DE TRANSFER}

- Desplazamiento disminuye $2 \%$

- Disminuye 70 metros de recorridos

- Disminuye 15 movimientos (ocasinados por traslado de la suela)

- Ganancia en tiempo de 5 minutos

\section{DISCUSIÓN}

Con los datos actuales del proceso se evidencian los múltiples problemas que se presentan. Observando la Figura 2 (VSM actual) y la Tabla 2 (PCE), la eficiencia se encontró un valor de $73.5 \%$, lo que indica que sobr- -epasa el 50\%, pero un valor aceptable sería alrededor de $80 \%$. También, de acuerdo al análisis del VSM se detectaron diferentes actividades que no aportan eficiencia al proceso, tales como un lead time de 21.37 horas y distancias que se deben de recorrer con un valor de 1371 metros. Con el conocimiento de los puntos débiles se obtiene un visión clara que permite implementar un plan acción.

Es así como, a través de una propuesta de acciones correctivas, se puede obtener un aumento de la eficiencia del $3.1 \%$, lo que significa que los tiempos de espera y los recorridos deben disminuir. Una vez identificadas y analizadas las oportunidades se procedió a visualizar los posibles resultados de las acciones de mejora. Cabe resaltar que no se omitió ningún proceso, con el análisis de los procesos de forma individual se genera una lluvia de ideas capaz de promover posibles cambios para encaminar el proceso a mejorar. Únicamente se realizaron mejoras dentro de los mismos procesos.

Entre las mejoras efectuadas tenemos las siguientes:

- Reducción de lead time

- Implementación de acciones correctivas

- Reducción de los desplazamientos

Analizando cada área que compone el proceso para realizar una chancla lámina Eva, se observa que el área de perforado es la única que no presenta inconvenientes a comparación de las demás, que sí presentan problemas con grandes distancias de desplazamiento, específicamente en tiempos que no agregan valor, por lo que se puede llegar a disminuir en tres de los cinco procesos que existen en la planta de Dj-Group.

Al realizar los cambios sugeridos, en la Figura 3 (VSM futuro) se evidencia una ganancia de tiempo en cuatro de las cinco áreas: inyección, troquel, transfer y ensamble. La mayor disminución está en el área de troquelado con una ganancia de 58 minutos, menor CO (cambios de operación), siguiendo el área transfer con 17 minutos, en el área inyectora se obtiene mayor disminución de OT (otros tiempos), 35 minutos en la variable de 
desplazamiento, el área con mayor decrecimiento en distancia es inyectora con el 13\%, seguido del área de troquelado disminuyendo un $9 \%$.

Respecto a la Figura 4 (comparativo de costo/ inversión), teniendo en cuenta que el total de pares utilizados para este ejercicio es de 5.040, se evidencia una mejora en el costo unitario $\$ 0.55$, con una diferencia en costo total que pasa de $\$ 15.384 .583,66$ a \$14.601.581; estas mejoras monetarias se hacen con base en la misma cantidad de trabajadores que se evidencian en el VSM actual al futuro, 19 operarios, pero las horas operativas se ven afectadas de acuerdo a los cambios implementados con una diferencia de 2.1 horas.

\section{CONCLUSIONES}

Al realizar el análisis a la empresa Dj-Group mediante la herramienta de Value Stream Mapping, que facilita el detalle paso a paso del proceso desde el proveedor hasta el cliente, se concluyeron los siguientes puntos:

- Es de vital importancia el conocimiento completo de cada proceso que influye en la creación de la chancla lámina Eva; la recolección de datos es un papel importante a la hora de poder medir y llegar a estandarizar las variables que comprende cada proceso. La importancia de conocer las actividades de los operadores atiende a que se pueden visualizar las diferentes fallas que se presentan, además de factores que no contribuyen a la creación de la chancla lámina Eva, por ejemplo: la acumulación de recorridos cortos pero repetitivos que influyen en los tiempos de ciclo (CT).

- De acuerdo a la información recolectada e implementación de un VSM para saber el estado actual del proceso, se pueden realizar varias técnicas con la finalidad de buscar solución a los problemas encontrados, como son: tiempo de espera y gran cantidad de recorrido; entre las técnicas que se pueden implementar para resolver los problemas encontrados está la lluvia de ideas, la cual nos permite obtener múltiples opiniones de cada problema existente y así desarrollar estrategias que logren reducir recorridos repetitivos y tiempos de espera innecesarios.

- De acuerdo con la herramienta utilizada (VSM), se pudo evidenciar las imperfecciones del proceso de la chancla lámina Eva para hombre, la cual arroja un lead time que perjudica la etapa productiva, es decir, el tiempo al desplazar la materia prima o en la búsqueda de implementos necesarios para el proceso, entre otros, incluso los recorridos que conllevan a esta actividad que pueden llegar a ser innecesarios. Con los datos obtenidos nos damos cuenta de que la eficiencia es de $76.6 \%$, la cual es baja, es necesario incrementarla, para así mismo disminuir los tiempos de espera y distancias recorridas.

- El VSM permitió mapear de manera general el proceso de fabricación de chancla en base a lámina EVA, identificando las mudas, en este caso os desplazamientos constantes y tiempos de alistamiento, que afectaban procesos de áreas claves para la entrega del producto final. Al respecto se obtuvo una disminución del $27 \%$ de desplazamientos generados por traslado de suela y lámina, se reduce 174,7 metros de distancias en las actividades mencionadas anteriormente, disminución también reflejada en los movimientos que se necesitan para hacer estos traslados, que equivalen a 115 movimientos de ganancia, lo que mejora este punto al aumentar la capacidad de docenas y en la parte de alistamiento una ganancia de 130 minutos al implementar las mejoras mencionadas en cada área. Estos tiempos se verán reflejados en mejorar la productividad de la compañía.

\section{REFERENCIAS BIBLIOGRÁFICAS}

American Society for Quality. (2012). ASQ. https://asq. org/quality-resources/lean/value-stream-map ping.

BAUDIN, M. (2013). ¿De dónde proviene los "Value Stream Maps"? https://michelbaudin.com/2013 /10/25/where-do-value-stream-maps-come-fro $\mathrm{m} /$ 
Clockwork. (2020). Clockwork. Obtenido de SMED: Una Inyección de Flexibilidad al Sistema Productivo:https://clockwork.com.co/ smed-una-inyeccion-de-flexibilidad-al-sistem a-productivo/

Ortega, F. (2021). Lean Manufacturing. Y Mayor Productividad en la Industria. https:// docplayer.es/3502495-Lean-manufacturing-ymayor-productividad-en-la-industria-el-entor no-actual-de-los-negocios-y-la-globalizacionhan-administracion.html

Leanopedia. (s.f.). Mapeo de flujo de valor: una guía sobre qué es y cómo usarlo. https:// leanopedia.com/value-stream-mapping-intro/

Terry, J. (2021). Planview. Obtenido de https://www. planview.com/resources/guide/lean-principle s-101/what-is-value-stream-mapping/

Sarria, M. P., Fonseca, G. A. y Bocanegra, C. C. (2017). Modelo metodológico de implementación de lean manufacturing. Revista EAN, 83, pp 51-71.https://doi.org/10.21158/01208160.n83. 2017.1825

\section{AUTORES}

Yaritza Jaramillo Barona: tecnóloga en gestión de la producción industrial SENA. Aspirante al título de ingeniero industrial de la UNIAJC. Integrante del semillero Simec. Correo: yaritzajb@hotmail.com

Richard Fernando Urbano Rayo: tecnólogo en gestión de la producción industrial SENA. Aspirante al título de ingeniero industrial de la UNIAJC. Integrante del semillero Simec. Correo: rfurban97@hotmail.com

Carlos Andres Nieto: ingeniero mecánico de la Universidad Autónoma de occidente, con estudios en automatización, gerencia financiera, MBA en admini- stración con énfasis en gerencia estratégica de la Universidad ICESI. Profesor tiempo completo de la facultad de ingeniería en la institución universitaria Antonio José Camacho, perteneciente al grupo de investigación Inteligo. Correo: canieto@admon.uniajc.edu.co 